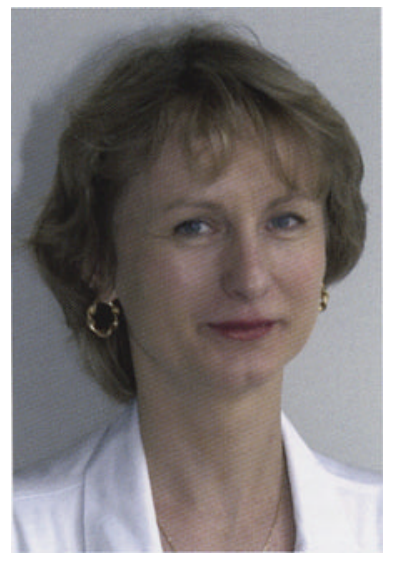

Par lo Dr Mirlam Gardner

Radiothérapeute, Radiotherapeute,
Centre René Huguenin de Lutte Contre le Cancer

"... La cancérologie, qui manque

de traitements efficaces,

est un des champs

de prédilection

de la recherche.

II s'agit de recherches très diversifiees, ciblèes aussi bien sur le profil génétique du patient que sur le diagnostic précoce des cancers, les facteurs

pronostiques qui pourraient guider

les traitements, les critères d'efficacitè

des traitements, les nouveaur traitements

cibles, le diagnostic de récidive précoce ..."

\section{La biologie médicale en cancérologie et la pratique quotidienne des cliniciens}

Les notions de biologie sont de plus en plus évoquées dans la pratique quotidienne des médecins. Certaines spécialités sont plus ou moins « exposées » à cette explosion de nouvelles découvertes faites en recherche biologique.

La cancérologie, qui manque de traitements efficaces, est un des champs de prédilection de la recherche. II s'agit de recherches très diversifiées, ciblées aussi bien sur le profil génétique du patient que sur le diagnostic précoce des cancers, les facteurs pronostiques qui pourraient guider les traitements, les critères d'efficacité des traitements, les nouveaux traitements ciblés, le diagnostic de récidive précoce; en bref, tout le champ de la cancérologie est largement étudié pour améliorer la prise en charge des patients et l'efficacité des traitements.

On sait tous que pour l'instant le traitement unique, efficace et sans effets secondaires en cancérologie n'a pas été découvert et peut-être, à notre grand regret, ne le sera jamais.

En attendant, est-ce que, nous, les cliniciens, nous tirons tous les renseignements possibles des résultats de recherche qui nous sont présentés? Par ailleurs, la recherche avance-t-elle au rythme le plus rapide possible, est-elle toujours faite de la façon la plus efficace pour répondre aux questions posées?

Je crains que la réponse soit non dans les deux cas.

Les cliniciens sont souvent submergés par une quantité de publications biologiques parfois obtenues avec un nombre limité de patients et contredites par des études ultérieures.

Les biologistes n'orientent pas toujours leur recherche de façon à obtenir des résultats dans des groupes homogènes et assez importants de patients.

Tout le monde aurait à gagner d'une collaboration plus étroite des cliniciens et des chercheurs, aussi bien dans le choix des thèmes et de la stratégie de la recherche proprement dite que dans l'application des données biologiques dans la pratique clinique.

Les cliniciens, par leur connaissance de la diversité de présentation clinique et d'évolution de la maladie cancéreuse, pourraient aider les chercheurs à créer des groupes cliniquement homogènes pour comparer ce qui est comparable, et aussi à inclure plus facilement des patients dans des études dont ils sont convaincus del'intérêt clinique.

Les biologistes peuvent apporter leur savoir pour interpréter au mieux les résultats des examens demandés en clinique. Un exemple flagrant entre tant d'autres est l'interprétation abusive d'une « positivité » d'un marqueur tumoral, sans prendre en compte les pathologies non tumorales pouvant être à l'origine d'une augmentation du marqueur, par simple ignorance de cette possibilité par le clinicien.

Certains produits de recherche biologique comme les marqueurs tumoraux sont largement utilisés en clinique, même si les modalités de leur utilisation optimale ne sont pas encore complètement définies.

D'autres avancées biologiques comme par exemple l'évaluation de la prédisposition génétique au cancer ont déjà une application clinique, même si l'on se doute qu'à l'avenir une place plus importante leur reviendra.

De plus, il y a des nouvelles voies de recherche biologique comme par exemple l'exploration des signatures métastatiques, dont l'intérêt est pour l'instant académique, même si on peut y voir un potentiel important d'applications cliniques futures.

On assiste aujourd'hui à la mise en place d'essais cliniques multiples qui étudient chacun une question particulière. Pourquoi ne pas imaginer d'adjoindre systématiquement à chaque essai thérapeutique une étude biologique, génétique ou pourquoi pas protéomique?

Actuellement en Europe, le fossé entre les cliniciens et les chercheurs reste encore trop large. Travailler ensemble plutôt qu'en parallèle pourrait nous aider à avancer plus vite et plus efficacement. 